\title{
Design of DuAl AXIS Solar TraCKER System BASED ON FUZZY INFERENCE SYSTEMS
}

\author{
Hamzah Hijawi and Labib Arafeh \\ Department of Computer Science, Arab American University, Jenin, Palestine
}

\begin{abstract}
Electric power is a basic need in today's life. Due to the extensive usage of power, there is a need to look for an alternate clean energy source. Recently many researchers have focused on the solar energy as a reliable alternative power source. Photovoltaic panels are used to collect sun radiation and convert it into electrical energy. Most of the photovoltaic panels are deployed in a fixed position, they are inefficient as they are fixed only at a specific angle. The efficiency of photovoltaic systems can be considerably increased with an ability to change the panels angel according to the sun position. The main goal of such systems is to make the sun radiation perpendicular to the photovoltaic panels as much as possible all the day times. This paper presents a dual axis design for a fuzzy inference approach-based solar tracking system. The system is modeled using Mamdani fuzzy logic model and the different combinations of ANFIS modeling. Models are compared in terms of the correlation between the actual testing data output and their corresponding forecasted output. The Mean Absolute Percent Error and Mean Percentage Error are used to measure the models error size. In order to measure the effectiveness of the proposed models, we compare the output power produced by a fixed photovoltaic panels with the output which would be produced if the dual-axis panels are used. Results show that dual-axis solar tracker system will produce $22 \%$ more power than a fixed panels system.
\end{abstract}

\section{KEYWORDS}

Fuzzy, Membership function, Universe of discourse, PV, ANFIS, DC motor, FLC.

\section{INTRODUCTION}

Fuzzy logic can be viewed as an extension of classical logical systems; the basic concept of the fuzzy set theory was first introduced by Zadeh in 1965 [1]. It provides an effective framework to deal with the problem of knowledge representation in an environment of uncertainty and imprecision. The concept of fuzzy logic is based on the degree of truth rather than the usual crisp Boolean values [2], it includes 0 and 1 as extreme cases of truth in addition to the various states between them. The importance of fuzzy logic was basically derived from the fact of human reasoning which are approximate values in nature. One of the main characteristics of the fuzzy models is the development of rules which relates the fuzzy input and the required output according to predefined membership functions [3]. A number of membership functions MF have been proposed in the past few years, namely the triangular, trapezoidal, Gaussian and bell shape functions. MF is defined as a graph that defines how each point in the input space is mapped to a value between 0 and 1 . The inputs are often referred as a universe of discourse which contains all the possible elements of concern [4]. 
The Adaptive Neuro-Fuzzy Inference System (ANFIS), developed in the early 90s by Jang in 1993 [5], that combines the concepts of fuzzy logic and neural networks to form a hybrid intelligent system to enhance the ability to automatically learn and adapt. Hybrid systems have been used by researchers for modeling and predictions in various engineering systems. The basic idea behind these neuro-adaptive learning techniques is to provide a method for the fuzzy modeling procedure to learn information [6].

In this paper, we present different types of fuzzy models for the dual-axis solar tracking system. The system is used to move photovoltaic panel according to the sun position in order to maximize the power produced. The system is modeled with different combinations of Mamdani fuzzy logic and adaptive neural fuzzy inference system ANFIS modeling, the testing data is compared with forecasted results using several metrics including: their Correlation Coefficients, The Mean Absolute Percent Error, MAPE, and the Mean Percentage Error, MPE errors.

The organization of the paper is as follow: we present different related works by other researchers in section two. Systems's deisgn is covered in section three. While several types of fuzzy models for the dual-axis solar tracking system are shown in sections four and five, section six discusses the results and section seven concludes the paper.

\section{RELATED WORK}

Fuzzy logic modeling has been widely used in many applications [4, 5]; fuzzy control is an example. However, fuzzy logic can be used to increase the output power of photovoltaic panels, sun tracking can increase the output power production by keeping the panel parallel to the sun, which makes the sun radiation perpendicular with the panel. Such system requires dual-axis tracking system. However, it was found that over 25 years' period PV dual-axis tracker will produce about 10 years' worth of additional solar energy [6,7]. In spite of the development of power electronics resources, the direct current machine became more and more useful. For example, the speed of DC motor can be adjusted to a great extent as to provide controllability easy and high performance [11]. Controllers can be in different types: PID Controller, Fuzzy Logic Controller; or the combination between them: Fuzzy-Neural Networks, Fuzzy-Genetic Algorithm. In [12], a MATLAB Simulink model for speed control of DC model using Mamdani fuzzy logic is provided, the simulation shows that the proposed fuzzy logic controller gives a smooth speed control with less overshoot and without oscillation. In [10], an application of ANFIS control for DC motor speed is presented. First, the system is modeled according to fuzzy rules, then an adaptive Neuro-Fuzzy controller of the DC motor speed is design and simulated. ANIFS has the advantage of the expert knowledge of the fuzzy inference system and the learning capability of the neural network. Results show that ANFIS models give better performance and high robustness than fuzzy rules alone.

In [13], a comparison is made for tuning methods of PID controllers to improve the performance of control system of DC motor. The system is first tuned using the conventional Zeigler method then the supervisory feature of fuzzy logic was used. The performance of the two approaches was compared, results show that the fuzzy logic approach has minimum overshoot, minimum transient, and steady-state parameters. [13] Provided a fuzzy logic control model in order to maximize the power point tracking algorithm for photovoltaic, the power variation and output voltage variation are used as inputs to the model. In order to improve the performance of the proposed method, the asymmetric membership function is used. Results show that FLC method can simultaneously shorten the tracking time and increase the tracking accuracy. 


\section{SYSTEM DESIGN}

The proposed system consists of a PV panel, four light sensors, two stepper motors. Sensors are placed in positions to read the sun illumination from the four directions; East, West, South, and North. One stepper motor is used to rotate the panel horizontally according to East and West light sensors readings, the other one is used to rotate the panel vertically according to South and North sensors readings as shown in figure 1 .

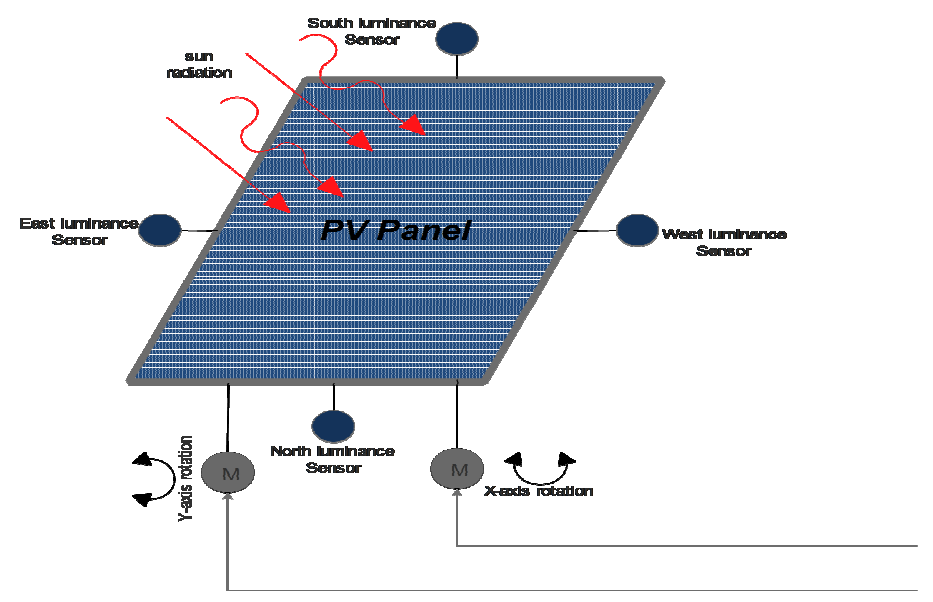

Figure1. PV dual axis panel

\section{FuZzY LOGIC MODELING}

The proposed model is based on the four light sensors readings inputs. Preprocessing phase takes the sensor readings as resistance values in ohm and converts them to a luminance scale between 0 to 1000 lux. Mamdani model is used to design the system. It is based on fuzzy inference system which employs the fuzzy if-then rules and can model the human knowledge and reasoning process without employing precise quantitative analysis [8]. A two-stage system is proposed as shown in figure 2, and the system model is described in figure 3.

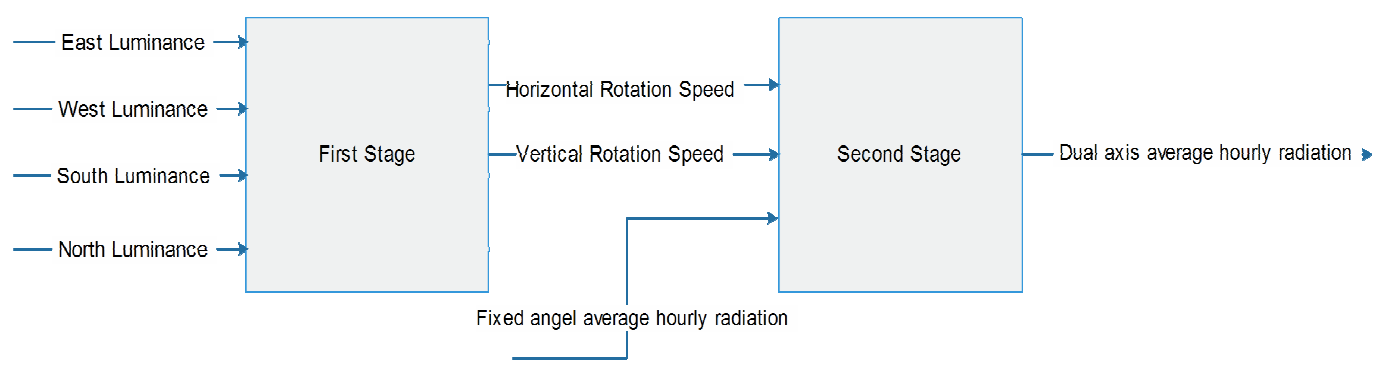

Figure 2. Proposed system block diagram 


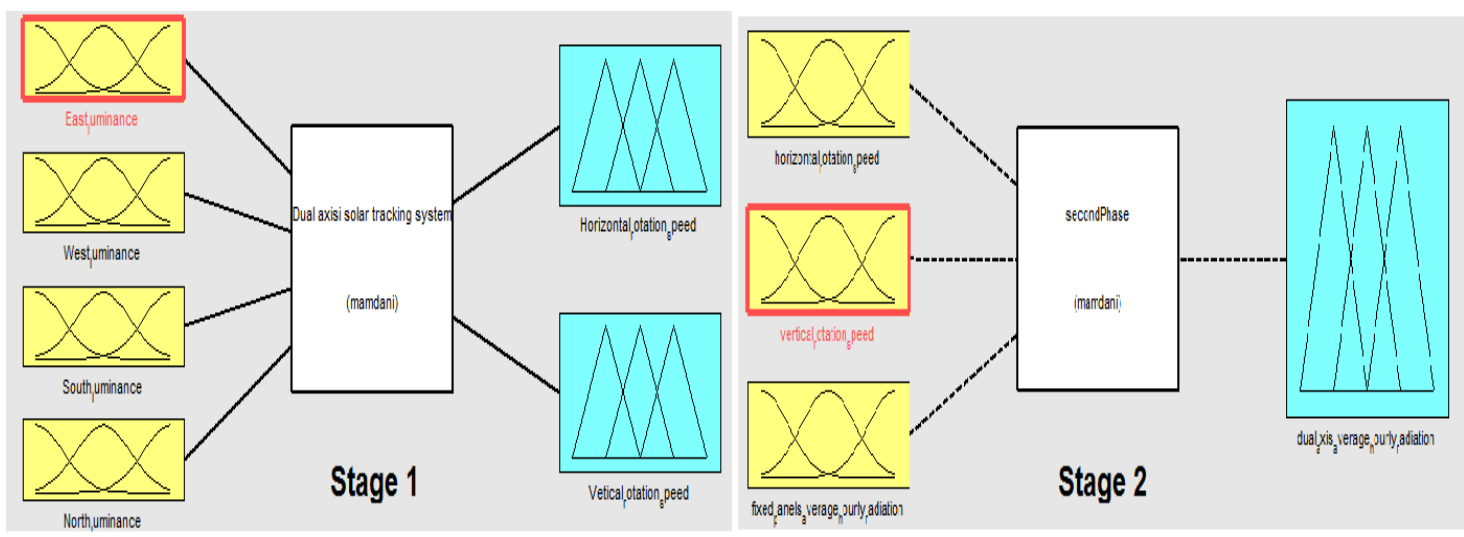

Figure 3. System model

East and West luminance values control the rotation speed and direction of the first motor in the horizontal axis while South and North values control the rotation speed and direction of the second motor in the vertical axis. The goal is to rotate the PV panel to a place which makes the sun radiation perpendicular to the PV panel, which in terms increases the total produced power. Trapezoidal member functions are used to describe the inputs and the outputs. Figure 4 shows the universe of discourse for each input, it has four membership functions; dim, dark, overcast and bright.

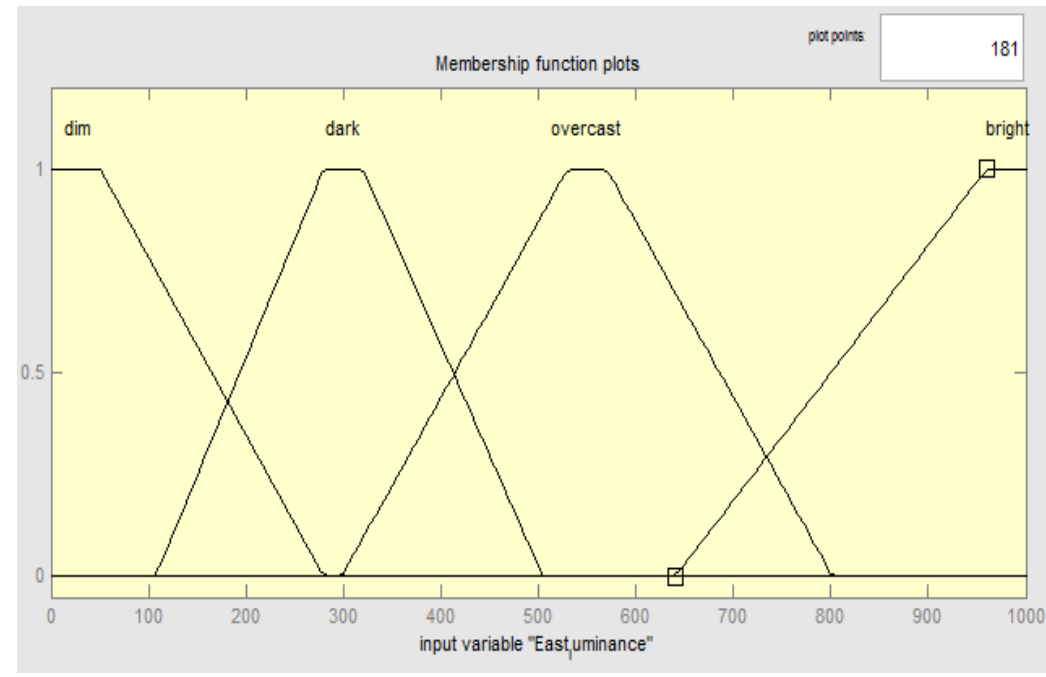

Figure 4. Inputs Membership functions

The output represents the rotation speed and direction of horizontal and vertical motors. Figure 5 shows the universe of discourse for each output, it has six trapezoidal membership functions in the range of -180 to 180 ; clockwise slow, clockwise mid, clockwise fast, counter clockwise slow, counter-clockwise mid and counter clockwise fast. 


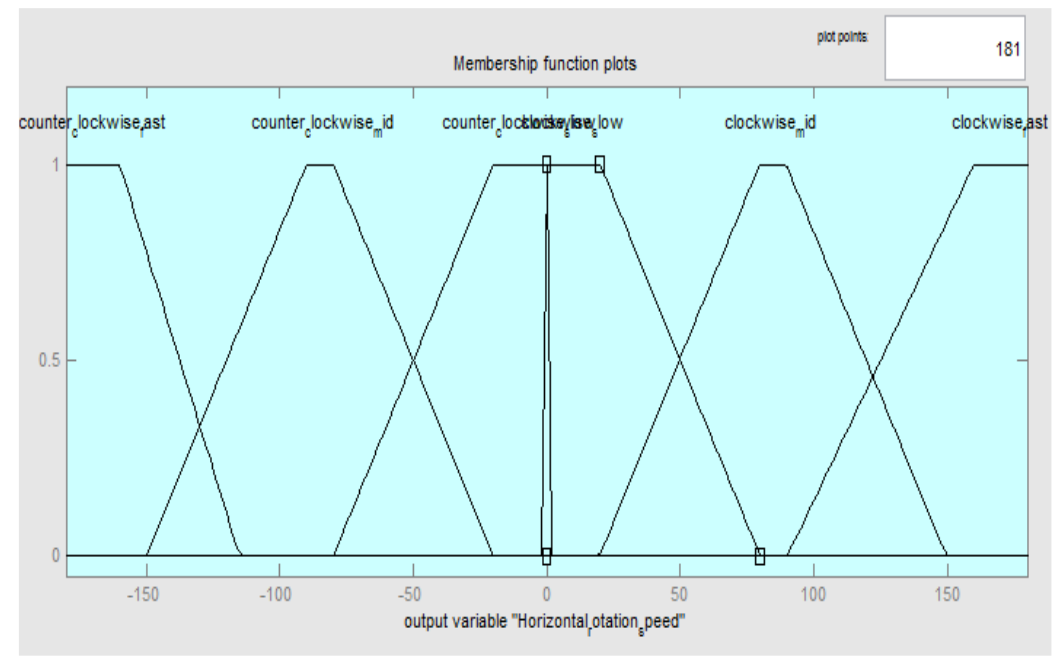

Figure 5. Output membership functions

Table 1 shows the fuzzy rules used to map east and west luminance values to a horizontal motor rotation speed and direction.

Table 1. Horizontal rotation rules

West Luminance

\begin{tabular}{|c|c|c|c|c|}
\hline & Dim & Dark & Overcast & Bright \\
\hline Dim & - & Clockwise slow & Clockwise mid & $\begin{array}{l}\text { Clockwise } \\
\text { fast }\end{array}$ \\
\hline Dark & $\begin{array}{c}\text { Counter clockwise } \\
\text { slow }\end{array}$ & - & Clockwise slow & $\begin{array}{c}\text { Clockwise } \\
\text { mid }\end{array}$ \\
\hline Overcast & $\begin{array}{l}\text { Counter clockwise } \\
\text { mid }\end{array}$ & $\begin{array}{c}\text { Counter clockwise } \\
\text { slow }\end{array}$ & - & $\begin{array}{l}\text { Clockwise } \\
\text { slow }\end{array}$ \\
\hline Bright & $\begin{array}{l}\text { Counter clockwise } \\
\text { fast }\end{array}$ & $\begin{array}{l}\text { Counter clockwise } \\
\text { mid }\end{array}$ & $\begin{array}{c}\text { Counter clockwise } \\
\text { slow }\end{array}$ & का \\
\hline
\end{tabular}

Rules in table 1 can be explained as follow:

1. If East luminance is dim and west luminance is dim then don't rotate.

2. If East luminance is dim and west luminance is dark then rotate clockwise with slow speed.

3. If East luminance is dim and west luminance is overcast then rotate clockwise with mid speed.

4. If East luminance is dim and west luminance is bright then rotate clockwise with fast speed.

5. If East luminance is dark and west luminance is dim then rotate counter clockwise with slow speed.

6. If East luminance is dark and west luminance is dark then don't rotate

7. If East luminance is dark and west luminance is overcast then rotate clockwise with slow speed. 
8. If East luminance is dark and west luminance is bright then rotate clockwise with mid speed.

9. If East luminance is overcast and west luminance is dim then rotate counter clockwise with mid speed.

10. If East luminance is overcast and west luminance is dark then rotate counter clockwise with slow speed.

11. If East luminance is overcast and west luminance is overcast then don't rotate.

12. If East luminance is overcast and west luminance is bright then rotate clockwise with slow speed.

13. If East luminance is bright and west luminance is dim then rotate counter clockwise with slow fast.

14. If East luminance is bright and west luminance is dark then rotate counter clockwise with mid speed.

15. If East luminance is bright and west luminance is overcast then rotate counter clockwise with slow speed.

16. If East luminance is bright and west luminance is bright then don't rotate.

The second motor which is responsible for vertical rotation is controlled by the other two inputs, south and north sensors. The rotation speed and direction of the motor is based on rules described in table 2 .

Table 2. Vertical rotation rules

South Luminance

\begin{tabular}{|c|c|c|c|c|}
\hline & Dim & Dark & Overcast & Bright \\
\cline { 2 - 6 } & - & $\begin{array}{c}\text { Counter clockwise } \\
\text { slow }\end{array}$ & $\begin{array}{c}\text { Counter clockwise } \\
\text { mid }\end{array}$ & $\begin{array}{c}\text { Counter } \\
\text { clockwise fast }\end{array}$ \\
\hline Dim & - & $\begin{array}{c}\text { Counter clockwise } \\
\text { slow }\end{array}$ & $\begin{array}{c}\text { Counter } \\
\text { clockwise mid }\end{array}$ \\
\hline Dark & Clockwise slow & - & - & $\begin{array}{c}\text { Counter } \\
\text { clockwise slow }\end{array}$ \\
\hline Bright & Clockwise fast & Clockwise mid & Clockwise slow & - \\
\hline
\end{tabular}

Rules in table 2 can be explained as follow:

1. If north luminance is dim and south luminance is dim then don't rotate.

2. If north luminance is dim and south luminance is dark then rotate counter clockwise with slow speed.

3. If north luminance is dim and south luminance is overcast then rotate counter clockwise with mid speed.

4. If north luminance is dim and south luminance is bright then rotate counter clockwise with fast speed.

5. If north luminance is dark and south luminance is dim then rotate clockwise slow.

6. If north luminance is dark and south luminance is dark then don't rotate.

7. If north luminance is dark and south luminance is overcast then rotate counter clockwise with slow speed.

8. If north luminance is dark and south luminance is bright then rotate counter clockwise with mid speed. 
9. If north luminance is overcast and south luminance is dim then rotate clockwise mid.

10. If north luminance is overcast and south luminance is dark then rotate clockwise slow.

11. If north luminance is overcast and south luminance is overcast then don't rotate.

12. If north luminance is overcast and south luminance is bright then rotate counter clockwise with slow speed.

13. If north luminance is bright and south luminance is dim then rotate clockwise fast.

14. If north luminance is bright and south luminance is dark then rotate clockwise mid.

15. If north luminance is bright and south luminance is overcast then rotate clockwise slow.

16. If north luminance is bright and south luminance is bright then don't rotate.

\section{ANFIS MODELING TECHNIQUES}

Adaptive neuro-fuzzy inference system ANFIS is a kind of artificial neural network that it is based on Sugeno fuzzy inference system [9]. ANFIS is a multilayer feedforward network which uses neural network learning algorithms and fuzzy reasoning to map an input space to an output space. With the ability to combine the verbal power of a fuzzy system with the numeric power of a neural system adaptive network, ANFIS has been shown to be powerful in modeling numerous processes.

The proposed system described in section 2 is also modeled with ANFIS modeling techniques. These are; grid partition without training, grid partition with hybrid training, grid partition with backpropagation training, subtractive clustering without training, subtractive clustering with hybrid training and subtractive clustering with backpropagation training. Grid partition divides the data space into rectangular subspaces using axis-paralleled partition based on a pre-defined number of membership functions and their types in each dimension [14]. The wider applications of grid partition in fuzzy logic and fuzzy inference systems are blocked by the curse of dimensions, which means that the number of fuzzy rules increases exponentially when the number of input variables increases. The subtractive clustering method clusters data points in an unsupervised way by measuring the potential of data points in the feature space. When there is not a clear idea how many clusters there should be used for a given data set, it can be used for estimating the number of clusters and the cluster centers [18]. Subtractive clustering assumes that each data point is a potential cluster center and calculates the potential for each data point based on the density of surrounding data points.

As discussed in section 2, the system has four inputs and two outputs, first and second inputs affect the results of the first output while the third and fourth inputs affect the results of the second output. Since ANFIS doesn't support multiple outputs, the system is divided into two single output models with two inputs for each one as shown in figure 6. 


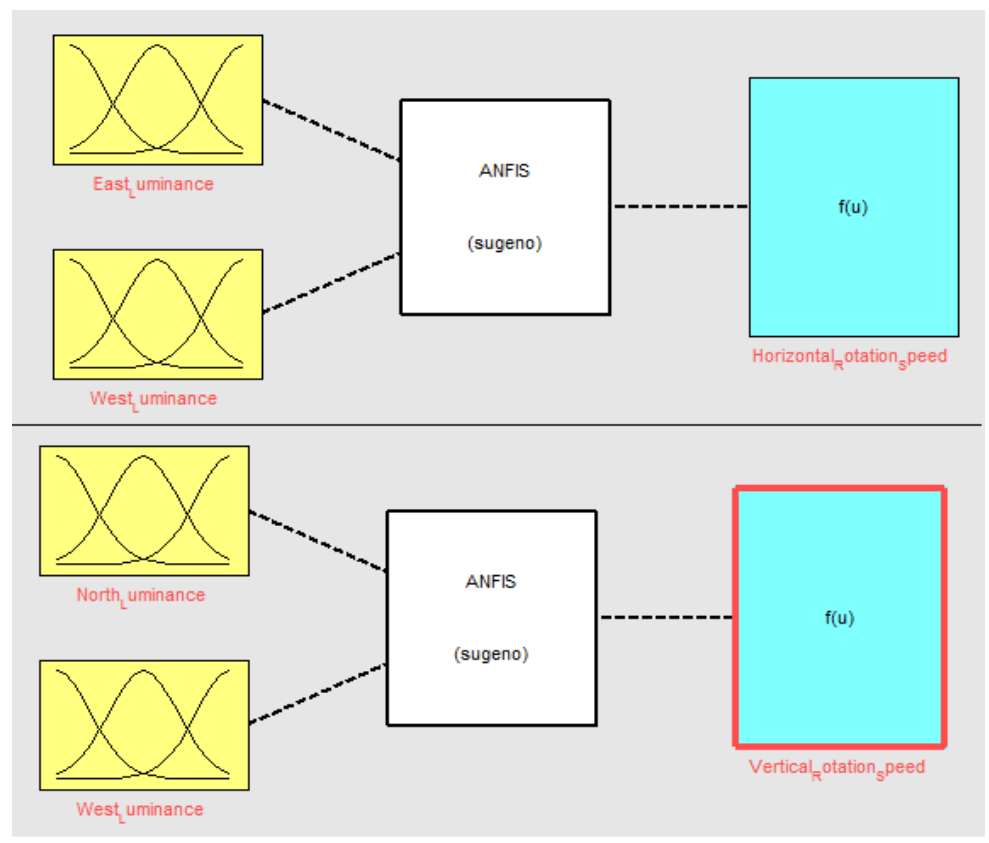

Figure 6. ANFIS system model

The number and the types of membership functions are the same as what is used in Mamdani model. For each input, the number of the membership functions is set to four and the type is set to trapmf. After generating FIS, the models structure will be as shown in figure 7. The figure shows the number of inputs, the number of membership functions for each input, the number of rules and the number of output membership functions.

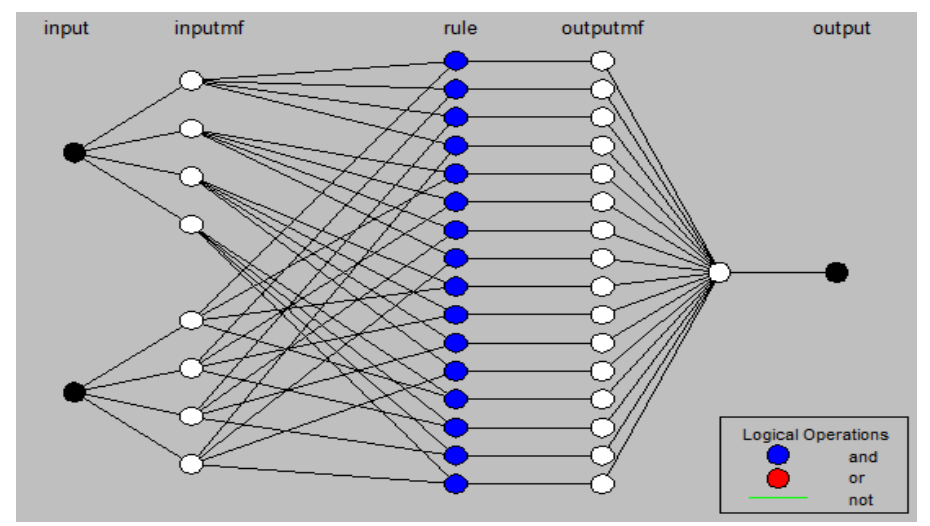

Figure 7. ANFIS model structure

To make the system operational, the models is trained with selected train datasets. Mainly, there are two optimum methods available for FIS training; hybrid and back propagation. Error tolerance is related to the error size and is used to decrease the average testing error and increase the system accuracy. Each model is trained with a dataset of $7 \mathrm{k}$ points. 


\section{RESULTS AND DISCUSSION}

In order to compare the accuracy of each model, a cross-validation testing with a dataset of $1.7 \mathrm{~K}$ points is used. Three comparison measures are used; correlation coefficient, MAPE, and MPE. A correlation coefficient is a statistical measure of the degree to which changes to the value of one variable predict change to the value of another. The Mean Absolute Percent Error MAPE is the most common measure of forecast error while Mean Percentage Error MPE is the computed average of percentage errors by which forecasts of a model differ from actual values of the quantity being forecast. Formulas 1, 2 and 3 show how each of these measures can be calculated. Results are summarized in table3.

$\operatorname{Corel}(X, Y)=\frac{\Sigma(x-\not x)(y-\bar{y})}{\sqrt{\varepsilon(n-\bar{x})^{2} \Sigma(y-\bar{y})^{2}}}$

MAPE $\%=\frac{100}{n} \sum \frac{|x-y|}{|x|}$

MPE $\%=\frac{100}{n} \sum \frac{x-y}{x}$

Where,

$\mathrm{X}$ : Actual results vector.

Y: Forecast results vector.

$\bar{x}$ : Mean of X.

$\bar{y}$ : Mean of $Y$.

Table 3. Testing measures comparison

\begin{tabular}{|c|c|c|c|c|}
\hline \multirow{2}{*}{ Model } & Output & $\begin{array}{c}\text { Correlation } \\
\mathbf{\%}\end{array}$ & $\begin{array}{c}\text { MAPE } \\
\mathbf{\%}\end{array}$ & $\begin{array}{c}\text { MPE } \\
\mathbf{\%}\end{array}$ \\
\hline \multirow{2}{*}{ Mamdani } & x_rotation & 98.28 & 6.84 & 5.21 \\
\cline { 2 - 5 } & y_rotation & 98.33 & 8.26 & 4.37 \\
\hline \multirow{2}{*}{ Grid Partition without training } & x_rotation & 0 & 48.74 & 100 \\
\cline { 2 - 5 } & y_rotation & 0 & 50.45 & 100 \\
\hline \multirow{2}{*}{$\begin{array}{c}\text { Grid Partition with hybrid } \\
\text { training }\end{array}$} & x_rotation & $\mathbf{9 8 . 7 7}$ & 1.53 & -7.78 \\
\cline { 2 - 5 } & y_rotation & $\mathbf{9 8 . 7 7}$ & 3.49 & -9.378 \\
\hline \multirow{3}{*}{$\begin{array}{c}\text { Grid Partition with backpropa } \\
\text { training }\end{array}$} & x_rotation & 98.73 & 2.03 & -7.88 \\
\cline { 2 - 5 } & y_rotation & 98.72 & 4.16 & -12.81 \\
\hline \multirow{2}{*}{$\begin{array}{c}\text { Clustering without training } \\
\text { Clustering with hybrid training }\end{array}$} & x_rotation & 98.60 & $\mathbf{1 . 0}$ & -4.70 \\
\cline { 2 - 5 } & y_rotation & 98.45 & 1.81 & -7.66 \\
\cline { 2 - 5 } & x_rotation & 98.60 & $\mathbf{1 . 0}$ & -4.70 \\
\hline \multirow{2}{*}{$\begin{array}{c}\text { Clustering with backpropa } \\
\text { training }\end{array}$} & y_rotation & 98.45 & 1.81 & -7.66 \\
\cline { 2 - 5 } & y_rotation & 98.60 & 1.67 & $\mathbf{- 2 . 2 7}$ \\
\cline { 2 - 5 } & y_rotation & 98.67 & 2.15 & -5.80 \\
\hline
\end{tabular}


As shown in table 3, the testing measures for all models are so close. However, the maximum correlation obtained is for grid partitioning with hybrid training while the minimum MAPE error is for clustering with and without training and the minimum MPE is for clustering with backpropagation model. Each model has its training method and this explains the small differ in values between all models. For the case of grid partitioning without training, the correlation between actual results and forecasted results is zero and the errors are at the maximum values, this is because the default training option for the grid modeling is without training. In such case, the system has not any previous knowledge about the testing data and is unable to recognize them. This is not the same for the case of cluster modeling, in which the default training option is hybrid. This explains the exact values obtained in clustering without training and with hybrid training.

Figures 8 a-g show plots comparison between the actual 1.7k data test points and their corresponding forecasted results. Plots show how the forecasted results are so close to the actual results.

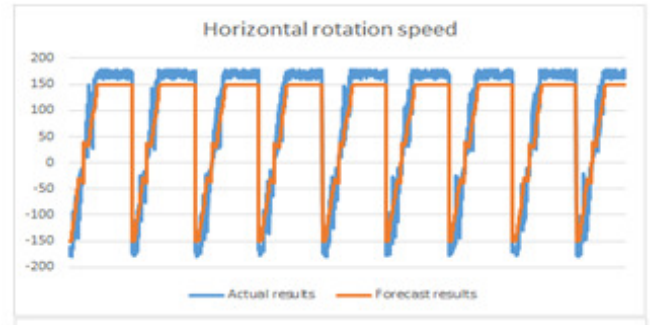

Vertical rotation speed

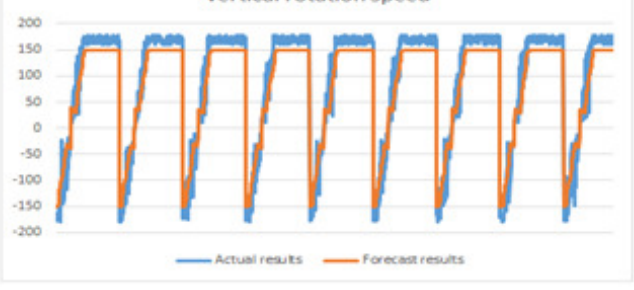

Figure 8(a). Mamdani results comparison

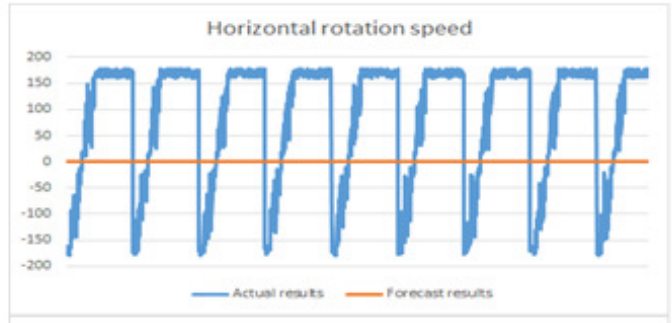

Vertical rotation speed

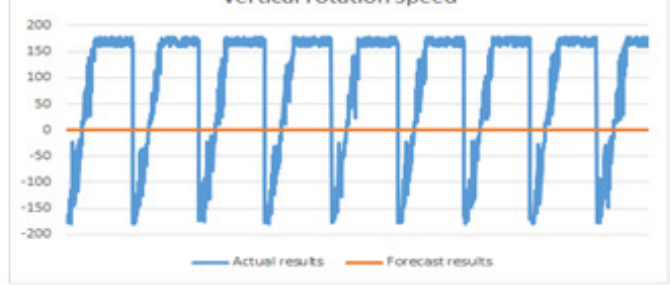

Figure 8 (b) ANFIS Grid partitioning without training 
International Journal on Soft Computing, Artificial Intelligence and Applications (IJSCAI), Vol.5, No.2/3, August 2016
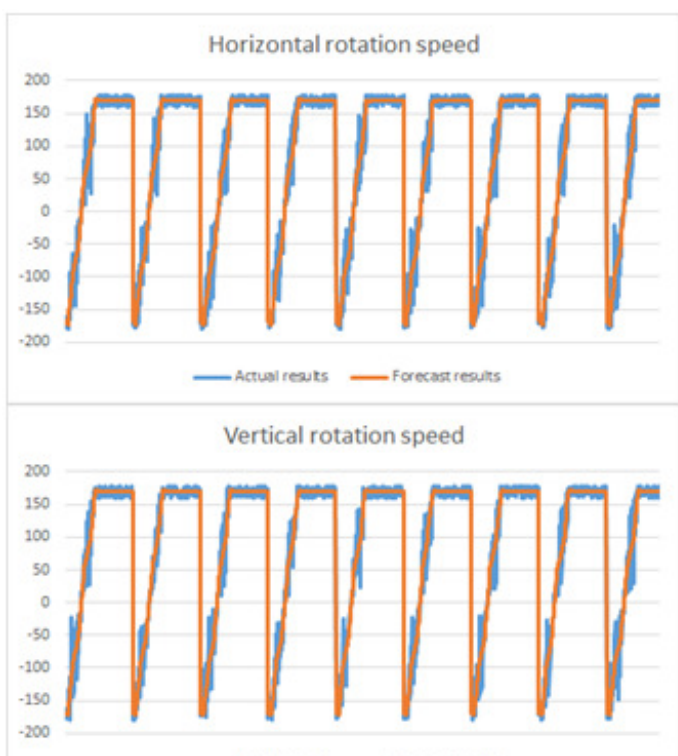

Figure 8 (c). ANFIS Grid partitioning with hybrid training

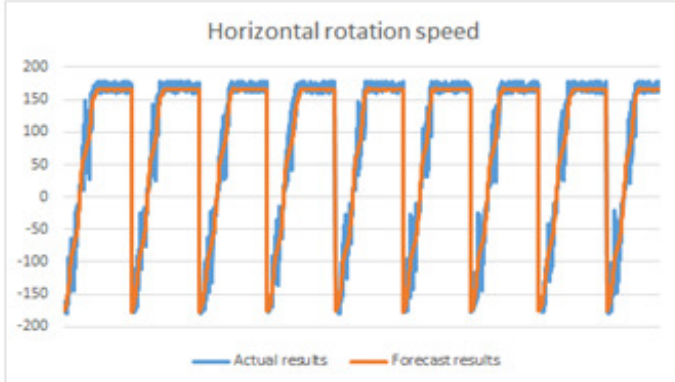

Vertical rotation speed

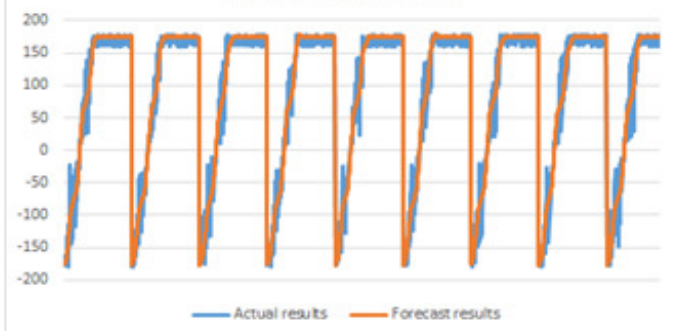

Figure 8 (d). ANFIS Grid partitioning with backprop a training

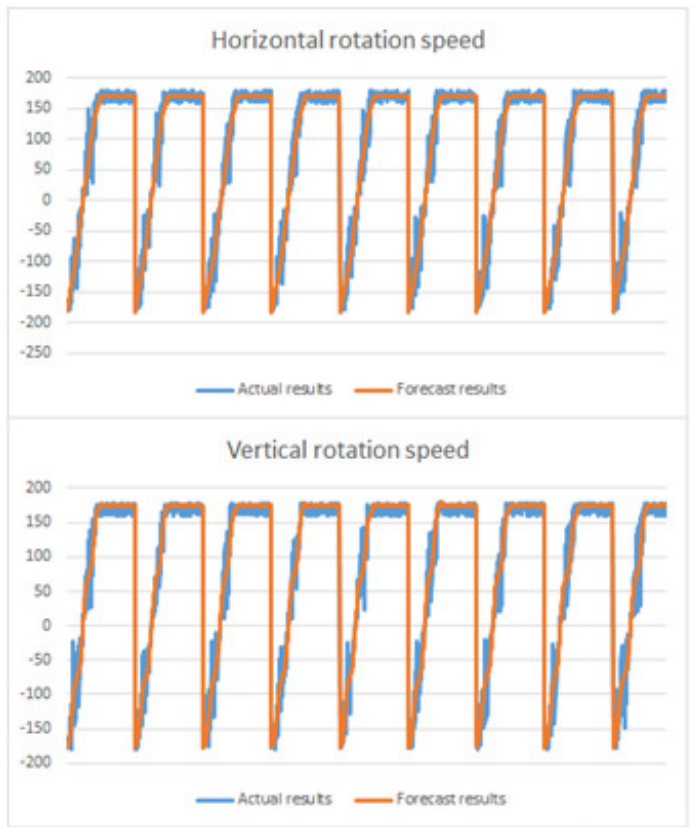

Figure 8 (e). ANFIS Clustering without training

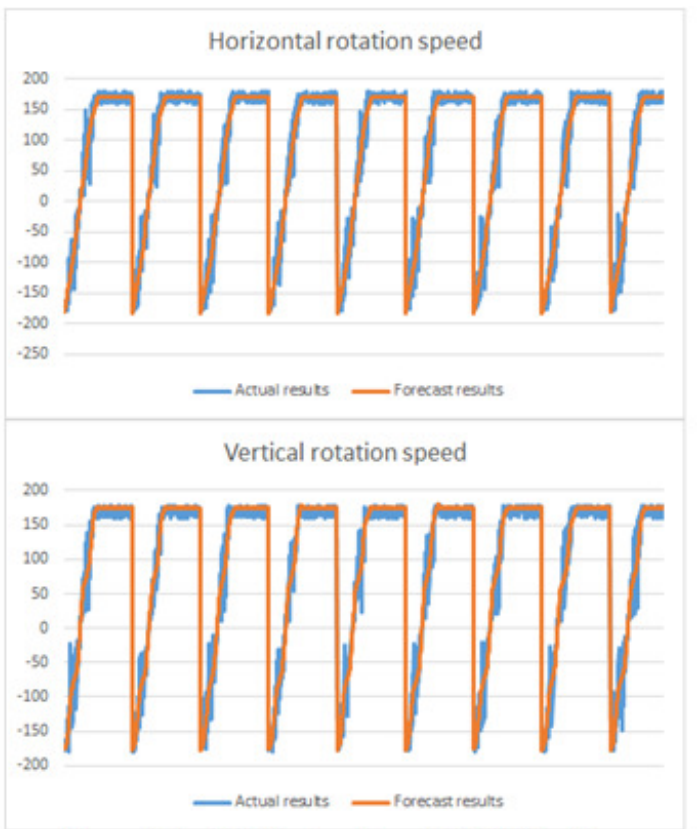

Figure 8 (f). ANFIS clustering with hybrid training 


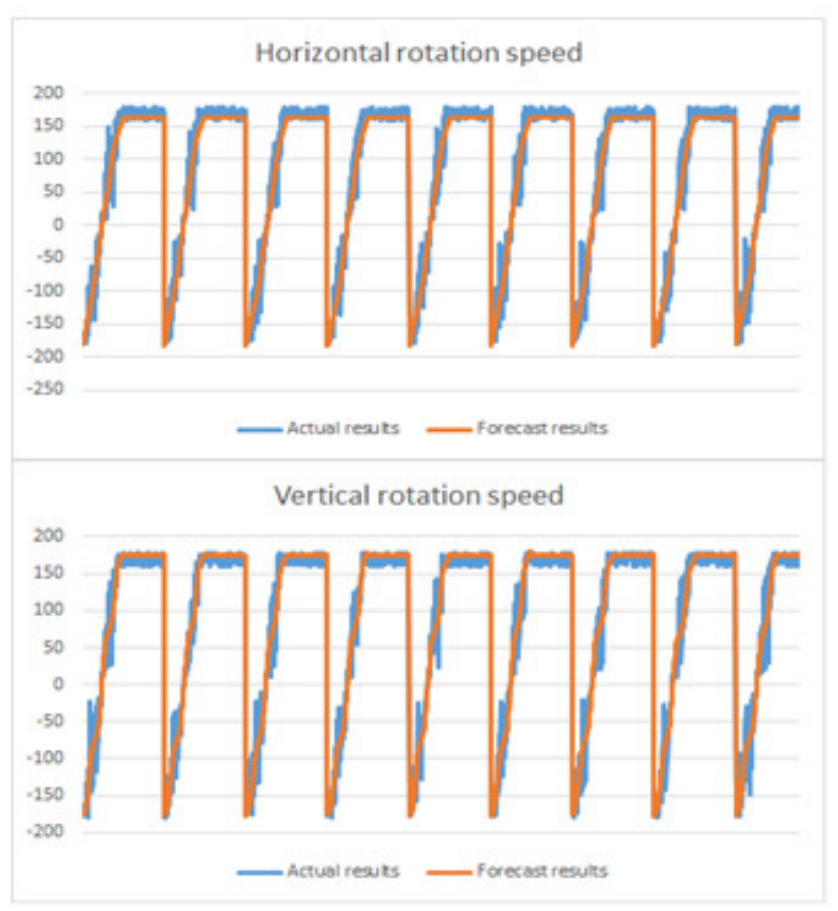

Figure 8 (g). ANFIS clustering with backpropa training

In order to show the effectiveness of the proposed models, we used the second stage of the proposed system model shown in figure 2 and applied ANFIS grid partitioning modeling with hybrid training option. The stage has three inputs, two of them are the outputs from the first stage. These are, horizontal_rotation_speed and vertical_rotation_speed, the third input is the real power produced by fixed axis PV system. The output of this stage represents the power which will be produced if the dual-axis solar tracker is used. In order to compare the actual power produced by a fixed photovoltaic system with the power produced by the dual-axis system proposed in this paper. The hourly average solar radiation data from the year of 2013 in Al-Najah solar panel system is used, results are summarized in table 4 and figure 9.

Table 1. Fixed and dual-axis power comparison

\begin{tabular}{|l|l|}
\hline Actual power in the fixed panels system W/m2 & 848595.7 \\
\hline Expected power in the dual-axis system W/m2 & 1039045.2 \\
\hline Gain & $22.44 \%$ \\
\hline & \\
\hline
\end{tabular}




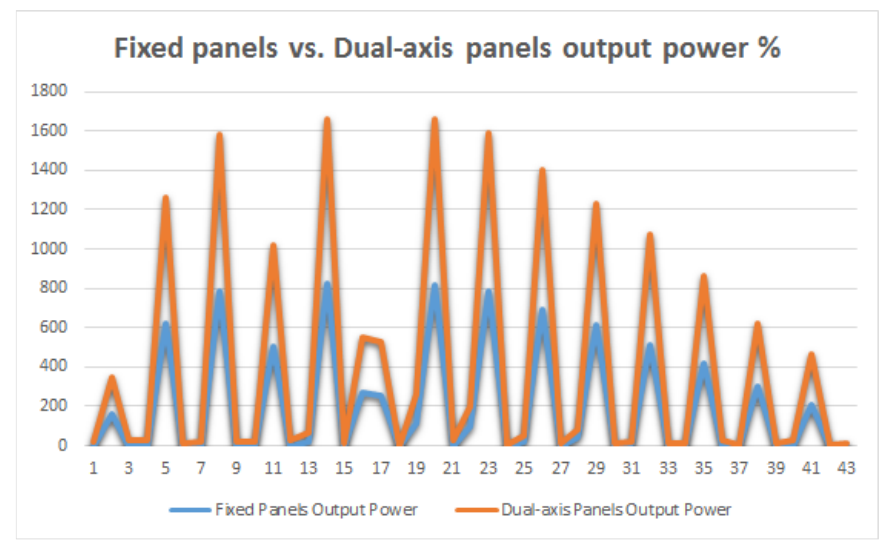

Figure 9. Fixed panels vs. Dual-axis output power \% for the year of 2013

\section{CONCLUSIONS}

In this paper many fuzzy logic models of dual-axis solar tracking systems were proposed and a fuzzy logic based DC motor speed control system was designed. First, the system was modeled using Mamdani fuzzy logic modeling then various cases of ANIFS models were applied. Same testing dataset is applied to each model and results were compared in terms of actual data correlation with forecasted data, MAPE and MPE error. In order to show the effectiveness of the proposed dual-axis solar tracking models, a second stage of fuzzy inference system was used to forecast the output power. Results from this model showed that the proposed dual-axis solar tracking system provided $22 \%$ more power than the fixed PV system.

\section{ACKNOWLEDGEMENTS}

We acknowledge that the data that is used in this comparison has been obtained from Al-Najah solar system in the year of 2013 [15].

\section{REFERENCES}

[1] Zadeh, Lotfi A. "Fuzzy sets." Information and control 8, no. 3 (1965): 338-353.

[2] Yager, Ronald R., and Lotfi A. Zadeh, eds. An introduction to fuzzy logic applications in intelligent systems. Vol. 165. Springer Science \& Business Media, 2012.

[3] Novák, Vilém, Irina Perfilieva, and Jiri Mockor. Mathematical principles of fuzzy logic. Vol. 517. Springer Science \& Business Media, 2012.

[4] Zhu, A-Xing, Lin Yang, Baolin Li, Chengzhi Qin, Tao Pei, and Baoyuan Liu. "Construction of membership functions for predictive soil mapping under fuzzy logic." Geoderma 155, no. 3 (2010): 164-174.

[5] Jang, J-SR. "ANFIS: adaptive-network-based fuzzy inference system." IEEE transactions on systems, man, and cybernetics 23, no. 3 (1993): 665-685.

[6] "PV Trackers Dual-Axis Benefits." PV Trackers Dual-Axis Benefits. Accessed May 11, 2016. http://www.pvtrackers.com/DualAxisBenefits.aspx.

[7] Rao, Roshan R., H. R. Swetha, J. Srinivasan, and Sheela K. Ramasesha. "Comparison of performance of solar photovoltaics on dual axis tracker with fixed axis at $13 \mathrm{~N}$ latitude." CURRENT SCIENCE 108 (2015): 1 . 
[8] Chai, Yuanyuan, Limin Jia, and Zundong Zhang. "Mamdani model based adaptive neural fuzzy inference system and its application." International Journal of Computational Intelligence 5, no. 1 (2009): 22-29.

[9] Chang, Fi-John, and Ya-Ting Chang. "Adaptive neuro-fuzzy inference system for prediction of water level in reservoir." Advances in Water Resources 29, no. 1 (2006): 1-10.

[10] Dastranj, Mohamad Reza, E. Ebroahimi, N. Changizi, and E. Sameni. "Control DC motorspeed with adaptive neuro-fuzzy control (ANFIS)."Australian Journal of Basic and Applied Sciences 5, no. 10 (2011): 1499-1504.

[11] Namazov, Manafeddin, and Onur Basturk. "DC motor position control using fuzzy proportionalderivative controllers with different defuzzification methods." Turkish Journal of Fuzzy Systems 1, no. 1 (2010): 36-54.

[12] Chakravorty, Jaydeep, and Ruchika Sharma. "Fuzzy logic based method of speed control of DC motor." international journal of emerging technology and advanced engineering 3, no. 4 (2013).

[13] Liu, Chun-Liang, Jing-Hsiao Chen, Yi-Hua Liu, and Zong-Zhen Yang. "An asymmetrical fuzzylogic-control-based MPPT algorithm for photovoltaic systems." Energies 7, no. 4 (2014): 2177-2193.

[14] Wei, Mingzhen, Baojun Bai, Andrew H. Sung, Qingzhong Liu, Jiachun Wang, and Martha E. Cather. "Predicting injection profiles using ANFIS."Information Sciences 177, no. 20 (2007): 4445-4461.

[15] "The MED-SOLAR Project Built a PV Power Plant in Palestine." An-Najah National University -. Accessed July 29, 2016. https://www.najah.edu/en/community/scientific-centers/scientificnews/2015/12/28/the-med-solar-project-built-a-pv-power-plant-in-palestine/.

\section{AUTHORS}

Hamzah Hijawi received his BS in computer system engineering from Birzeit University, Ramallah, Palestine, in 2011. He is currently working with Exalt technologies as a software engineer since 2010, and currently pursuing his Master of Computer Science from Arab American University, Jenin, Palestine. His researches interest include Computer Networks, Information Security, Wireless Sensor Networks and Data Mining.

Labib Arafeh has over Thirty years of professional experience including Information Technology applications, teaching, training, administration, development and planning, tied with hands-on exposure monitoring, evaluation \& supervisory responsibilities. Dr. Arafeh has obtained his expertise from working experience at three universities, study visits and as the director of the National Accreditation \& Quality Assurance Commission, as well leading and participating in developing and implementing several local and global related projects. He has also been involved in managing, supervising and implementing several international \& local projects such as developing e-Learning and quality assurance

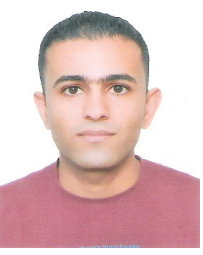
policies for EMUNI university. Dr. Arafeh has been involved in leading and participating in related several international \& local projects including the UNESCO funded IT \& Electrical Engineering Benchmarks, RAND (US)-Al-Quds University funded effective teaching project, EU-supported FINSI, ICT-LEAP and RUFO Tempus projects. In addition, Dr. Arafeh has participated in evaluating \& studying positive and negative impacts of several World Bank \& EU proposed projects. Also, he evaluated the technology 5-10 school curricula and developed the textbook for the 8th grade. Furthermore, Dr. Arafeh's main research topics including Applying Neurofuzzy modeling techniques to applications like: Water and Power Load Demand Predictions, Management Key Knowledge Areas, Entrepreneurship Key Competencies, etc., quality eLearning systems, Multimedia, Automatic Essay Grading and Scoring, mobile Text-To-Speech, and Software Engineering. 